\title{
Structural Differences within Negative and Depressive Syndrome Dimensions in Schizophrenia, Organic Brain Disease, and Major Depression: A Confirmatory Factor Analysis of the Positive and Negative Syndrome Scale
}

\author{
Daniel P. Eisenberg ${ }^{a} \quad$ Dmitry B. Aniskin ${ }^{a} \quad$ Leonard White $^{b} \quad J_{\text {Judith A. Stein }}^{c}$ \\ Philip D. Harvey ${ }^{d}$ Igor I. Galynker ${ }^{a}$ \\ a Department of Psychiatry, Beth Israel Medical Center, New York, N.Y., ${ }^{b}$ Clinical Neuroscience Center, Pilgrim \\ Psychiatric Center, Brentwood, N.Y., ' Department of Psychology, University of California, Los Angeles, Calif., and \\ ${ }^{\mathrm{d} D e p a r t m e n t}$ of Psychiatry, Emory University, Atlanta, Ga., USA
}

\section{Key Words}

Confirmatory factor analysis · Major depression • Negative

symptoms $\cdot$ Schizophrenia $\cdot$ Organic brain disease

\begin{abstract}
Objective: The emerging dimensional approach to classification and treatment of psychiatric disorders calls for better understanding of diagnosis-related variations in psychiatric syndromes and for proper validation of psychometric scales used for the evaluation of those syndromes. This study tested the hypothesis that negative and depressive syndromes as measured by the Positive and Negative Syndrome Scale (PANSS) are consistent across different diagnoses. Method: We administered the PANSS to subjects with schizophrenia $(n=305)$, organic brain disease (OBD, $n=66$ ) and major depressive disorder (MDD, $\mathrm{n}=75$ ). Confirmatory factor analysis (CFA) was used to establish if the PANSS items for negative symptoms and for depression fit the hypothesized factor structure and if the item factor loadings were similar among the diagnostic groups. Results: The negative and depressive symptom subscales fit well according to a variety of fit indexes for all groups individually after some modest model
\end{abstract}

modification. However, multisample modeling procedures indicated that the pattern of factor loadings was significantly different among the groups in most cases. Conclusion: The results of this study indicate diagnosis-related variations in the negative and depressive syndrome dimensions in schizophrenia, OBD and MDD. These results also validate limited use of the PANSS for evaluation of negative and depressive syndromes in disorders other than schizophrenia. Larger studies are warranted to further evaluate clinical and nosologic significance of diagnostic categories, dimensions and structures of psychiatric syndromes.

Copyright $\odot 2009$ S. Karger AG, Basel

\section{Introduction}

Negative and depressive syndromes have been described in the psychiatric literature as both independent of one another [1] and present in several major neuropsychiatric disorders [2-6]. Although classically considered an essential element of schizophrenia [7], the negative syndrome has been reported in major depression [1], stroke [4, 5], and dementia [3]. Similarly, Hafner et al. [8]

\section{KARGER}

(c) 2009 S. Karger AG, Basel

Fax +41613061234 E-Mail karger@karger.ch www.karger.com www.karger.com/psp
Igor I. Galynker, $\mathrm{MD}, \mathrm{PhD}$

Department of Psychiatry, Beth Israel Medical Center

First Avenue \& 16th Street

New York, NY 10003 (USA)

Tel. +1 212420 4535, Fax +1 212844 7659, E-Mail igalynke@chpnet.org 
showed that the depressive syndrome occurs not only in affective illness, but also in the vast majority of subjects with prodromal schizophrenia. Furthermore, the depressive syndrome is also common in organic brain diseases (OBDs), such as stroke [9] and dementia [10].

The presence of similar negative and depressive syndromes in different diagnostic settings has also encouraged hypotheses about the neurobiological 'final common pathway' of the depressive syndrome, which often feature disturbances of striatal and frontal circuitry $[9$, $11]$.

If the negative and depressive syndromes represent valid cross-diagnostic dimensions reflecting common underlying functional abnormalities in the brain, then they should retain similar forms in different diagnostic settings. Preliminary evidence based on the use of exploratory factor analysis of the Positive and Negative Symptom Scale (PANSS) in heterogeneous populations of outpatients with major depressive disorder (MDD) [12] and OBD [13] supports this notion. These promising data are tempered by a study of schizophrenic and bipolar patients in acute psychotic episodes, which found that exploratory factor analysis of PANSS cluster scores in these groups yielded differences in both the negative and depressive factor structures [14]. This discrepancy highlights the need for more precise approaches for the assessment of the stability of the negative and depressive syndromes across diagnoses.

Although our study is not the first to explore the hypothesis of cross-diagnostic syndromal dimensions, we are aware of no confirmatory factor analyses (CFA) that compare negative and depressive syndromes in individuals with different neuropsychiatric disorders, except for one report demonstrating that a five-factor model, including both negative and depressive syndrome factors, had the highest goodness of fit index for a mixed population of subjects with schizophrenia, delusional disorder and bipolar disorder [15]. This model is similar to many five-factor solutions in factor analytic studies of schizophrenic subjects $[16,17]$. Thus, the results of the study indirectly provide further support for a dimensional approach to psychiatric diagnosis, which includes negative and depressive syndromes.

To determine how robust the negative and depressive syndromes are in different diagnostic settings, we used CFA of data obtained by administering PANSS to subjects with schizophrenia, OBD and major depression. We hypothesized that we would find a model that fits well for both syndromes in all groups and that both syndromes would not differ significantly among the groups.

\section{Materials and Methods}

Subjects

Three subject cohorts were studied: patients with schizophrenia, MDD and OBD.

\section{Subjects with Schizophrenia}

A total of 305 elderly (mean age 75.7 years, $\mathrm{SD}=7.0,44 \%$ male) poor-outcome inpatients with a lifetime research diagnosis of schizophrenia were studied. A full description of the diagnostic procedure is presented in Davidson et al. [18]. Patients met DSMIII-R criteria based upon the lifetime medical record, interviews with collateral informants and the patients themselves. Exclusion criteria were: a diagnosis of schizophreniform and schizoaffective disorder, history of mental retardation, active treatment of a seizure disorder, presence of neurological disorder, or severe sensory deficit. A minimum of physical, neurological and routine laboratory tests were performed on all patients no more than 1 year prior to assessment.

Symptom assessment was carried out as part of the patients' clinical care and data were obtained from the medical record. Data were used only if raters judged patients to be sufficiently cooperative and cognitively intact for valid interviews. The mean Mini Mental State Exam score in the elderly poor-outcome schizophrenic subjects was 15 .

\section{Subjects with OBD}

Subjects with OBD consisted of patients with dementia $(\mathrm{n}=$ $45)$, including dementia of the Alzheimer type (DAT) $(n=17)$ and vascular or mixed dementia $(n=28)$, and stroke patients $(n=31)$ who were recruited from the Beth Israel Medical Center, New York, USA. These diagnoses were combined into one group largely because they are all primary brain diseases with gross or microscopic brain lesions that may give rise to associated negative and depressive symptoms $[4,13,19]$, as well as for the sake of additional statistical power. Due to some missing data, 66 subjects were used in the current study. OBD subjects were $44-94$ years of age (mean age $72.6, \mathrm{SD}=12.34,57 \%$ male). The inclusion criteria for the diagnostic groups were dementia and stroke.

Dementia. Subjects were 45 dementia patients who showed clinical signs of negative symptoms and were recruited from consecutive admissions to medical wards at the Beth Israel Medical Center in New York City. Typically, the subjects were admitted for treatment of infection, were already medically stable at the time of this evaluation, and were awaiting placement when enrolled in this study. All patients were under the care of an internist, a neurologist and a psychiatrist, and all underwent a full neurological evaluation including either CT or MRI of the head. The selection criteria used were similar to the ones described by Sultzer et al. [20]. Seventeen patients met the National Institute of Neurological and Communicative Disorders and Stroke and the Alzheimer's Disease and Related Disorders Association Work Group (NINCDS-ADRDA) criteria for DAT. Twenty-eight presented with clinical dementia, did not meet NINCDS-ADRDA criteria for DAT [21], had histories of hypertension and showed vascular lesions on CT or MRI scans. Their likely diagnosis was vascular dementia, although in the absence of postmortem histological data, they may have had a combination of vascular dementia and DAT. Patients with acute medical problems, current symptoms of agitation, or symptoms of psychosis were excluded. Toxic, meta- 
bolic, and infectious causes of dementia were ruled out by clinical examination and laboratory data. Patients' thyroid function tests, vitamin $B_{12}$ and folate levels were within normal limits, and the rapid plasma reagin test (syphilis test) was negative. Some patients had histories of agitation or depression and were receiving psychotropic medications, antipsychotics and/or antidepressants. Those who were not agitated or significantly depressed (Hamilton Depression Rating Scale score $\geq 14$ ) at the time of the intake evaluation were included. None of the subjects had preexisting histories of schizophrenia or substance abuse. The mean Mini Mental State Exam score for subjects with dementia was 17.63.

Stroke. Thirty-one nonaphasic stroke patients were evaluated 3-14 days after the stroke. CT confirmed stroke location in all patients. Strokes were localized as follows: left parietal $(\mathrm{n}=5)$; right parietal or temporoparietal $(n=7)$; right frontal $(n=2)$; left basal ganglia $(n=7)$; right basal ganglia $(n=5)$; midbrain $(n=1)$; pons $(n=4)$. Patients with histories of DSM-III-R axis I psychiatric disorder, Parkinson's disease, or other major central nervous system disorders were excluded. Specifically, none of the patients included in the study met DSM-III-R criteria for major depression. Stroke patients with possible preexisting dementia were included in the study. The mean Mini Mental State Exam score for subjects with stroke was 24.17 .

\section{Subjects with MDD}

Subjects were 75 patients admitted to acute psychiatric units of the Beth Israel Medical Center in New York for treatment of MDD. The average age of patients with MDD was 44.53 (SD = $10.15,37 \%$ male). All patients fulfilled DSM-IV diagnostic criteria for MDD as determined by 2 attending psychiatrists examining the patients independently. All patients gave written informed consent. They all had routine lab tests (e.g. CBC/PLT/Diff, SMA22, $\mathrm{U} / \mathrm{A}, \mathrm{RPR})$, were treated with either antidepressants or electroconvulsive therapy and were discharged from the inpatient units with at least satisfactory response. No patient with MDD included in this study received antipsychotics. All patients were evaluated with the 17-item Hamilton Depression Rating Scale prior to being scored on the PANSS. Interviews and rating scales were administered and scored by medical doctors and master's level graduate students in psychology, supervised by 2 of the authors.

\section{Assessment}

All subjects with schizophrenia, OBD, and MDD were administered the PANSS. The PANSS is a 30 -item scale, which includes a 7-item positive scale, a 7-item negative scale, and a 16-item general psychopathology scale that includes ratings of disturbances of mood. Items are rated on a 7-point scale. The PANSS is validated for schizophrenia and is based upon a patient interview and caretaker report. The current study uses the 7-item negative scale and the 5-item depression scale (negative: blunted affect, emotional withdrawal, poor rapport, apathetic social withdrawal, lack of spontaneity, mannerisms, motor retardation; depressive: anxiety, tension, depression, guilt, somatic concern).

All raters were trained using videotaped interviews and achieved satisfactory interrater reliability in three ratings (kap$\mathrm{pa}=0.80)$. Interrater reliability was determined separately in all three groups. Additionally, the reliability of ratings in the geriatric schizophrenic patients was evaluated in 54 ratings by paired observers at the same interview. The item interclass correlation were uniformly high (interclass correlation coefficient $=0.86-1.00$ ).

\section{Goodness of Fit of the PANSS Model}

CFA was used to evaluate two criteria essential to establishing the equivalency of factors between groups. First, the adequacy of a proposed factor model as indicated by various goodness of fit indexes is separately established in each group. Secondly, a between-group comparison is performed to determine if item factor loadings are essentially similar between groups [22].

The present study used the syndrome models described by White et al. [17] with minor modifications. This was the best-fitting model derived from a sample of patients with schizophrenia, including ambulatory and institutionalized geriatric and nongeriatric patients. The model described in the original study applied to five components taken together and used a complex model with some items having a secondary factor loading. Secondary factor loadings were not used in the present study because we were considering separate PANSS components but not the entire model. The negative syndrome and the depressive syndrome were examined separately.

\section{Statistical Analysis}

The data were analyzed using the EQS Structural Equation Modeling Program [23]. Fit was assessed with the comparative fit index (CFI), the maximum likelihood $\chi^{2}$, and the standardized root mean square residual (SRMR). CFI values of 0.95 or greater are desirable and, in the case of the SRMR, a small value indicates a well-fitting model. Values of less than 0.08 are desirable. We also report the reliability for each scale within each group.

Reliability of findings in structural equation modeling, as in principal CFA, may be undermined by the small sample size. The recommended minimum ratio of cases to parameters in the model is $5: 1$, but $10: 1$ is preferable [23]. Modeling only the primary factor loadings using separate small models with relatively few parameters helped mitigate the small sample sizes in the current study. Because the sample sizes were marginal for the OBD and MDD cohorts, if the single sample modeling of a syndrome indicated a good fit, we planned to use a bootstrap procedure to test the stability of the final parameter estimates [24]. One hundred and twenty separate samples were randomly derived from the specified cohort and the final model was run 120 times using each derived sample [25]. Ninety-five percent confidence intervals were computed for factor loadings and error terms in the final model after the parameters obtained from the 120 analyses were averaged across the randomly selected samples. Sample parameters within the $95 \%$ confidence limits determined by the bootstrap replications indicate stability of the findings.

Identification of a well-fitting model in each group is insufficient for establishing the invariance of the factor structure between groups. If a good fit was obtained in single-sample modeling and stability of parameters was supported by bootstrap sampling, two-group multisample comparisons were calculated with the schizophrenia cohort as the reference group. One model constrained item factor loadings to be equal between groups; the other, unconstrained, model left item factor loadings free to vary between the groups. In multiple-sample analyses, the $\chi^{2}$ difference between constrained and unconstrained models, based on the difference in degrees of freedom, tests for equivalence of key parameters between the samples (i.e., factor loadings). If the $\chi^{2}$ difference is statistically significant then all parameters are not reasonably equal and the groups differ in their relations between the factor and the individual measured variables. 
Table 1. Factor loadings and fit indexes for each group in the CFA models

\begin{tabular}{|c|c|c|c|}
\hline Variables & $\begin{array}{l}\text { OBD } \\
\text { factor } \\
\text { loadings }\end{array}$ & $\begin{array}{l}\text { MDD } \\
\text { factor } \\
\text { loadings }\end{array}$ & $\begin{array}{l}\text { Schizo- } \\
\text { phrenia } \\
\text { factor } \\
\text { loadings }\end{array}$ \\
\hline \multicolumn{4}{|l|}{ Negative syndrome } \\
\hline Blunted affect & 0.83 & 0.92 & 0.61 \\
\hline Emotional withdrawal & 0.92 & 0.95 & 0.75 \\
\hline Poor rapport & 0.85 & 0.96 & 0.76 \\
\hline Apathetic social withdrawal & 0.76 & 0.89 & 0.72 \\
\hline Lack of spontaneity & 0.92 & 0.98 & 0.88 \\
\hline Mannerisms & 0.29 & 0.82 & 0.40 \\
\hline Motor retardation & 0.83 & 0.90 & 0.45 \\
\hline \multicolumn{4}{|l|}{ Fit indexes } \\
\hline ML $\chi^{2} /$ d.f. & $33.73 / 13$ & $42.01 / 13$ & $27.05 / 13$ \\
\hline CFI & 0.95 & 0.97 & 0.99 \\
\hline SRMR & 0.06 & 0.02 & 0.03 \\
\hline Reliability coefficient & 0.93 & 0.98 & 0.85 \\
\hline \multicolumn{4}{|l|}{ Depression } \\
\hline Somatic concern & 0.53 & 0.93 & 0.45 \\
\hline Anxiety & 0.59 & 0.95 & 0.54 \\
\hline Guilt & 0.45 & 0.95 & 0.35 \\
\hline Tension & 0.35 & 0.94 & 0.46 \\
\hline Depression & 0.86 & 0.92 & 0.65 \\
\hline \multicolumn{4}{|l|}{ Fit indexes } \\
\hline$\chi^{2} /$ d.f. & $5.99 / 4$ & $15.59 / 5$ & $14.49 / 4$ \\
\hline CFI & 0.96 & 0.98 & 0.97 \\
\hline SRMR & 0.06 & 0.01 & 0.04 \\
\hline Reliability coefficient & 0.74 & 0.97 & 0.62 \\
\hline
\end{tabular}

All factor loadings are significant: $\mathrm{p} \leq 0.001 . \mathrm{ML}=$ Maximum likelihood.

\section{Results}

The results of the single-sample models of negative symptoms and depressive symptoms for the schizophrenia, OBD and MDD samples are summarized in table 1. All fit indexes are excellent and all factor loadings are significant ( $\mathrm{p} \leq 0.001$ ). One correlated error residual was added for all groups between the residuals for emotional withdrawal and apathetic social withdrawal in the negative syndrome scale. For the depression scale, a correlated error residual was added for the OBD group (anxiety and guilt) and the schizophrenia groups (anxiety and tension). No correlated error residuals were necessary for the depression group on the depression scale which, not surprisingly, had the highest factor loadings on the scale of the 3 groups. All reliability coefficients were satisfactory
Table 2. Results of multiple-group analyses comparing schizophrenia, OBD, and MDD groups

\begin{tabular}{|c|c|c|}
\hline & $\chi^{2}$, d.f. & $\begin{array}{l}\text { CFI, } \\
\text { SRMR }\end{array}$ \\
\hline \multicolumn{3}{|c|}{ Negative syndrome } \\
\hline \multicolumn{3}{|c|}{ Schizophrenic vs. OBD } \\
\hline Model 1 & $60.77,26$ & $0.97,0.05$ \\
\hline Model 2 & $\begin{array}{l}91.96,32 \\
\text { difference }^{1}: 31.19,6 \\
(p \leq 0.001)\end{array}$ & $0.96,0.16$ \\
\hline \multicolumn{3}{|c|}{ Schizophrenic vs. MDD } \\
\hline Model 1 & $69.05,26$ & $0.98,0.03$ \\
\hline Model 2 & $\begin{array}{l}125.53,32 \\
\text { difference } 1: 56.48,6 \\
(p \leq 0.001)\end{array}$ & $0.95,0.12$ \\
\hline \multicolumn{3}{|c|}{ Depression } \\
\hline \multicolumn{3}{|c|}{ Schizophrenic vs. OBD } \\
\hline Model 1 & $20.50,8$ & $0.97,0.05$ \\
\hline Model 2 & $\begin{array}{l}26.51,12 \\
\text { difference }^{1}: 6.01,4 \\
\text { (nonsignificant) }\end{array}$ & $0.97,0.07$ \\
\hline \multicolumn{3}{|c|}{ Schizophrenic vs. MDD } \\
\hline Model 1 & $30.08,9$ & $0.98,0.03$ \\
\hline Model 2 & $\begin{array}{l}63.83,13 \\
\text { difference }^{1}: 33.75,4 \\
(\mathrm{p} \leq 0.001)\end{array}$ & $0.95,0.08$ \\
\hline
\end{tabular}

Model 1: unconstrained measurement, model 2: constrained measurement.

${ }^{1}$ Difference between model 2 and model 1.

and indicate the reasonableness of using these syndrome scales in these patient groups. Supplementary bootstrap simulations for both the negative syndrome and depression models found item factor loadings, fit indexes, and error terms within $95 \%$ confidence limits for the smaller samples suggesting the reliability of parameters for these symptoms.

Table 2 summarizes the results of the multisample models contrasting the schizophrenia group versus the OBD and MDD groups, respectively. The $\chi^{2}$ differences in most cases were statistically significant indicating that the patterns of item factor loadings were not the same in these samples. Only the contrast between the schizophrenia group and the OBD group for the depression scale was nonsignificant. 


\section{Discussion}

The present results establish the applicability of the PANSS negative syndrome model to schizophrenia, OBD, and MDD. Similarly, the PANSS depressive syndrome model described our data well in all diagnostic groups. Because almost all between-group comparisons of the PANSS item factor loadings were significantly different, it is likely that neither the negative nor depressive syndromes in these different diagnostic settings are identical; however, the differences between the OBD and other groups could also be attributed to the insufficient size of the OBD group.

Although it is one of the most widely used psychometric instrument in schizophrenia, the PANSS is infrequently applied to nonschizophrenic psychiatric subjects [12-14]. The results of the present study indicate that the PANSS is a valid instrument for the assessment of the negative syndrome in schizophrenia, MDD, and OBD. These findings are consistent with previous studies establishing the negative syndrome as an independent dimension in Alzheimer's dementia and stroke [5, 13]. Furthermore, these data seem to be compatible with research of Marin [25] who has proposed a syndrome of apathy, which comprises symptoms almost identical to the negative syndrome [11], and, similarly, has been described in schizophrenia [26], Alzheimer's dementia [27], and Parkinson's disease [19]. However, to what degree particular data from the apathy literature are applicable here must wait quantitative research clarifying the relationship between these two sister constructs.

The findings of the present study suggest that a single instrument may be appropriate for the assessment of a syndrome across diagnostic groups even though expression of the syndrome may not be identical across the groups. One possibility for these differences may be due to limitations of the PANSS itself. Another possibility is that although the syndrome appears to be the same between diagnostic groups there are subtle but real differences. Our findings of subtle differences in the structure of negative symptoms between patients with schizophrenia and MDD are consistent with the findings of Kulhara and Chadda [2]. They reported higher scores on the Scale for the Assessment of Negative Symptoms on ratings of subjective complaints, suggesting greater insight in MDD patients whereas patients with schizophrenia scored higher on alogia, poor eye contact, inappropriate affect, and blocking. Of note, Daneluzzo et al. [14] reported that 'lack of insight' loaded on the negative syndrome factor in schizophrenic subjects but not in bipolar subjects. Per- haps then, the negative syndrome in affective illness is colored by greater cognitive status (e.g. capacity for selfappraisal), resulting in altered expression of select negative symptoms relative to negative symptoms of schizophrenia.

Of interest, the Hamilton Depression Rating Scale [28], validated and widely used for the assessment of depressive symptoms in MDD, was reported to be inappropriate for such use because of significant overlap of depressive symptoms with both positive and negative symptoms [29]. In contrast, our results demonstrate that the PANSS, although not designed for this specific purpose, can be used for evaluation of depression in both disorders, as well as in stroke and dementia, and retrospectively supports previous work using the PANSS this way $[5,6]$.

Thus, in validating the limited use of the PANSS to evaluate both the negative syndrome and the depressive syndrome in schizophrenia, OBD and MDD, we have demonstrated that existing psychometric scales can be used for dimensional evaluation of psychiatric syndromes, even across diverse diagnostic groups. However, in light of current trends toward more dimensional approaches to psychiatric diagnosis, researchers and clinicians should be cautious. For example, the differences that were demonstrated between the structure of PANSS ratings of the negative syndrome in MDD and schizophrenia reinforce the importance of scale validation prior to use in any given population. Because a single instrument may be appropriately used to assess a syndrome across different diagnostic groups does not in itself indicate that the syndromes are the same in the two groups.

Furthermore, the differences between diagnostic groups in the factor structure of both the negative and depressive syndromes seem to justify the current categorical boundaries between the studied diagnostic groups. Our results are consistent with the findings of other authors, according to which depressive and negative syndromes in various psychiatric disorders are clinically different and, hence, derive from different neurobiological mechanisms. For example, in schizophrenia, previous research has found a significant correlation between depressive symptoms and psychosis severity [30] or positive symptoms [31]. Additionally, depressive symptoms frequently remit with antipsychotic treatment alone [32], and outcomes of pharmacotherapy for depression in schizophrenia have been mixed [33]. These reports, although not probative, are consistent with the hypothesis that in schizophrenia, depression may be an epiphenomenon of psychosis and borne of different neuropathology 
than in primary depression. This is supported by work by Abel et al. [34], which demonstrated that administration of D-fenfluramine, a serotonergic agonist, is associated with decreased prolactin in MDD and increased prolactin in schizophrenia. Thus, among these past studies and our own here, there remains no clear evidence that the depressive syndrome arises from similar neurobiological substrates in these two disorders.

The present results need to be considered in light of its limitations. First, because of statistical power considerations, the group of subjects with organic brain pathology was heterogeneous, including both patients with $\mathrm{Alz}$ heimer's dementia and those with stroke. Therefore reported differences in syndrome structure between subjects with OBD and the other two groups may have resulted from lack of diagnostic uniformity. On the other hand, this nonconformity only underscores the validity of the PANSS for evaluation of negative and depressive syndromes in patients with OBD. Second, as the mean age of the schizophrenia group is 75.7, this group does not represent a random sample of patients with schizophrenia. However, we think that in our sample of elderly, poor-outcome schizophrenic patients, most of which were in the residual stage of their illness at the time of this study, both depressive and negative symptoms were stabilized and were representative of the genuine schizophrenic process. There is evidence to indicate that in elderly poor-outcome patients with schizophrenia the structure of PANSS items is independent of severity of cognitive impairment [35] and is not significantly different than the symptom structure in younger chronic patients with schizophrenia [17]. Autopsy studies conducted in the same cohort of elderly patients used in the present study find that severity of cognitive impairment is not related to the pathology of Alzheimer's or other known dementias [36]. Third, the sample size was relatively small for the OBD and MDD cohorts, and, although the bootstrap procedure is suggestive of the reliability of results, a larger sample size would be more conclusive and corroborative. Next, the differences in depressive syndrome structure can be attributed to the limitations of the PANSS itself, as, according to several studies, it has questionable validity in the assessment of depressive symptoms in schizophrenia $[37,38]$. However, our results met the criteria for a well-fitting model (i.e., CFI greater than 0.95, SRMR less than 0.08) in all three groups, reflecting the appropriateness of the PANSS for the assessment of depressive symptoms in MDD, OBD and schizophrenia. The MDD group in particular had excellent fit statistics validating the usefulness of the de- pressive symptom subscale among patients with major depression.

Last, our study was limited to the analysis of PANSS scores in schizophrenia, OBD, and MDD. Therefore, although we believe the results of this study have general implications for the current trend toward dimensional diagnostic system development, they must be interpreted as limited to this particular scale and these three neuropsychiatric illnesses. Nevertheless, the disorders investigated in this study are common and represent three major groups of severe psychiatric illnesses (psychotic, affective and cognitive), which increases the importance of the study.

In summary, within its limitations, this is the first study that validates the limited use of the PANSS for evaluation of depressive and negative symptoms in disorders other than schizophrenia. Additionally, in schizophrenia, MDD, and OBD, both negative and depressive syndromes as described by the PANSS are structurally unique, supporting the value of current categorical boundaries, indicating different neurobiological mechanisms and cautioning against treating patients solely based on these syndromes without considering the diagnostic context. We believe larger studies are warranted to validate our findings in broader populations and to further evaluate the relationship between diagnostic categories and structures of psychiatric syndromes.

\section{Acknowledgements}

This work was supported in part by funds from the National Institute of Mental Health (grant No. 950MH66392-01, a Conte Center Grant awarded to Kenneth M. Davis, MD), and The National Institute on Drug Abuse (grant No. DA 01070-34 awarded to J.A. Stein).

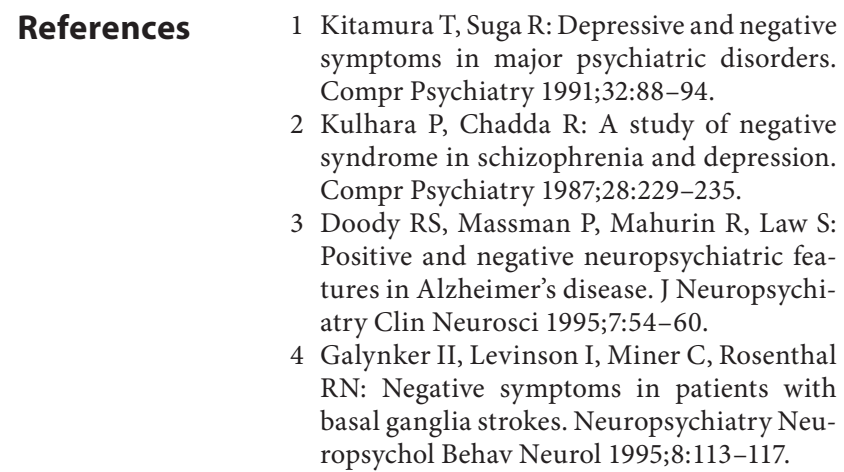

Psychopathology 2009;42:242-248 
5 Galynker I, Prikhojan A, Phillips E, Focseneanu M, Ieronimo C, Rosenthal R: Negative symptoms in stroke patients and length of hospital stay. J Nerv Ment Dis 1997;185:616621.

6 Galynker II, Dutta E, Vilkas N: Hypofrontality and negative symptoms in patients with dementia of Alzheimer type. Neuropsychiatry Neuropsychol Behav Neurol 2000;13:5359.

7 Jackson H: Remarks on evolution and dissolution of the nervous system. J Ment Sci 1894; 33:25-48.

$\checkmark 8$ Hafner H, Mauer K, Trendler G, Van der Heiden W, Schmidt M, Konnecke R: Schizophrenia and depression: challenging the paradigm of two separate diseases - a controlled study of schizophrenia, depression and healthy controls. Schizophr Res 2005;77:1124.

D9 Whyte EM, Mulsant BH: Post-stroke depression. Biol Psychiatry 2002;52:253-264.

-10 Zubenko GS, Zubenko WN, McPherson S, Spoor E, Marin DB, Farlow MR, Smith GE, Geda YE, Cummings JL, Petersen RC, Sunderland T: A collaborative study of the emergence and clinical features of the major depressive syndrome of Alzheimer's disease. Am J Psychiatry 2003;160:857-866.

11 Brown RG, Pluck G: Negative symptoms: the 'pathology' of motivation and goal-directed behaviour. Trends Neurosci 2000;23:412417.

$\checkmark 12$ Purnine DM, Carey KB, Maisto SA, Carey MP: Assessing positive and negative symptoms in outpatients with schizophrenia and mood disorders. J Nerv Ment Dis 2000;188: 653-661.

-13 Milak MS, Aniskin DB, Eisenberg DP, Prikhojan A, Cohen LJ, Yard SS, Galynker II: The negative syndrome as a dimension: factor analyses of PANSS in major depressive disorder and organic brain disease compared with negative syndrome structures found in the schizophrenia literature. Cogn Behav Neurol 2007;20:113-120.

-14 Daneluzzo E, Arduini L, Rinaldi O, Di Domenico M, Petruzzi C, Kalyvoka A, Rossi A: PANSS factors and scores in schizophrenic and bipolar disorders during an index acute episode: a further analysis of the cognitive component. Schizophr Res 2002;56:129136.
15 Serretti A, Olgiati P: Dimensions of major psychoses: a confirmatory factor analysis of six competing models. Psychiatry Res 2004; 127:101-109.

16 Kay SR, Sevy S: Pyramidal model of schizophrenia. Schizophr Bull 1990;16:537-545.

17 White L, Harvey PD, Opler L, Lindenmayer JP: Empirical assessment of the factorial structure of clinical symptoms in schizophrenia. A multisite, multimodel evaluation of the factorial structure of the Positive and Negative Syndrome Scale. The PANSS Study Group. Psychopathology 1997;30:263-274.

18 Davidson M, Harvey PD, Powchik P, Parrella M, White L, Knobler HY, Losonczy MF, Keefe RS, Katz S, Frecska E: Severity of symptoms in chronically institutionalized geriatric schizophrenic patients. Am J Psychiatry 1995;152:197-207.

19 Aarsland D, Litvan I, Larsen JP: Neuropsychiatric symptoms of patients with progressive supranuclear palsy and Pakinson's disease. J Neuropsychiatry Clin Neurosci 2001; 13:42-49.

20 Sultzer DL, Levin HS, Mahler ME, High WM, Cummings JL: A comparison of psychiatric symptoms in vascular dementia and Alzheimer's disease. Am J Psychiatry 1993; 150:1806-1812.

21 McKhann G, Drachman D, Folstein M Katzman R, Price D, Stadlan EM: Clinical diagnosis of Alzheimer's disease: report of the NINCDS-ADRDA Work Group under the auspices of Department of Health and $\mathrm{Hu}-$ man Services Task Force on Alzheimer's Disease. Neurology 1984;34:939-944.

22 Stein JA, Lee JW, Jones PS: Assessing crosscultural differences through the use of multiple group invariance analysis. J Pers Assess 2006;87:249-258.

23 Bentler PM: EQS Structural Equations Program Manual. Encino, Multivariate Software, 2006.

$24 \mathrm{Hu}$ LT, Bentler PM, Kano Y: Can test statistics in covariance structure analysis be trusted? Psychol Bull 1992;112:351-362.

25 Marin RS: Apathy: a neuropsychiatric syndrome. J Neuropsychiatry Clin Neurosci 1991;3:243-254.

26 Roth RM, Flashman LA, Saykin AJ, McAllister TW, Vidaver R: Apathy in schizophrenia: reduced frontal lobe volume and neuropsychological deficits. Am J Psychiatry 2004; 161:157-159.

-27 Benoit M, Clairet S, Koulibaly PM, Darcourt J, Robert PH: Brain perfusion correlates of the apathy inventory dimensions of $\mathrm{Alz}$ heimer's disease. Int J Geriatr Psychiatry 2004; 19:864-869.
28 Hamilton M: A rating scale for depression. J Neurol Neurosurg Psychiatry 1960;23:5662.

29 Addington D, Addington J, Atkinson M: Reliability and validity of a depression rating scale for schizophrenics. Schizophr Res 1992;6:201-208.

30 Zisook S, McAdams LA, Kuck J, et al: Depressive symptoms in schizophrenia. Am J Psychiatry 1999;156:1736-1743.

-31 Emsley RA, Oosthuizen PP, Joubert AF, Roberts MC, Stein DJ: Depressive and anxiety symptoms in patients with schizophrenia and schizophreniform disorder. J Clin Psychiatry 1999;60:747-751.

32 Koreen AR, Siris SG, Chakos M, Alvir J, Mayerhoff D, Lieberman J: Depression in first-episode schizophrenia. Am J Psychiatry 1993;150:1643-1648

33 Micallef J, Fakra E, Blin O: Use of antidepressant drugs in schizophrenic patients with depression. Encephale 2006;32:263269.

34 Abel KM, O'Keane V, Murray RM, Cleare AJ: Serotonergic function and negative and depressive symptomatology in schizophrenia and major depression. Psychoneuroendocrinology 1997;22:539-548.

35 White L, Harvey PD, Parrella M, Sevy S, Powchik P, Davidson M: Empirical assessment of the factorial structure of clinical symptoms in schizophrenic patients: symptom structure in geriatric and nongeriatric samples. New Trends Exp Clin Psychiatry 1994;12:75-83.

-36 Purohit DP, Perl DP, Haroutunian V, Powchik P, Davidson M, Davis KL: Alzheimer disease and related neurodegenerative diseases in elderly patients with schizophrenia: a postmortem neuropathologic study of 100 cases. Arch Gen Psychiatry 1998;55:205211.

>37 Kim SW, Kim SJ, Yoon BH, Kim JM, Shin IS, Hwang MY, Yoon JS: Diagnostic validity of assessment scales for depression in patients with schizophrenia. Psychiatry Res 2006; 144:57-63.

38 Van den Oord EJ, Rujescu D, Robles JR, Giegling I, Birrell C, Bukszár J, Murrelle L, Möller HJ, Middleton L, Muglia P: Factor structure and external validity of the PANSS revisited. Schizophr Res 2006;82:213-223. 